\title{
2-Aminooxazole as a Novel Privileged Scaffold in Antitubercular Medicinal Chemistry
}

Elisa Azzali, ${ }^{a, b,}$, Miriam Girardini,,${ }^{a, b}$ Giannamaria Annunziato, ${ }^{a, b}$ Marialaura Pavone, ${ }^{a, b}$ Federica Vacondio, ${ }^{b, d}$ Giorgia Mori, ${ }^{c}$ Maria Rosalia Pasca, ${ }^{c}$ Gabriele Costantino,,${ }^{a, b, d, e}$ Marco Pieroni, ${ }^{a, b, d^{*}}$ ${ }^{a}$ P4T group, and ${ }^{b}$ Department of Food and Drug, University of Parma, 43124 Parma, Italy; ${ }^{c}$ Department of Biology and Biotechnology "Lazzaro Spallanzani”, University of Pavia, 27100 Pavia; ${ }^{d}$ Centro Interdipartimentale "Biopharmanet-tec", Università degli Studi di Parma, Parma, Italy; ${ }^{e}$ Centro Interdipartimentale Misure (CIM) 'G. Casnati', University of Parma, Parma, Italy.

${ }^{¥}$ Current Address: Aptuit (Verona) Srl, an Evotec Company, Via Alessandro Fleming, 4, 37135 Verona, Italy

\section{Contents}

1. Experimental and general procedures $\quad$ S2

2. Determination of the MIC $\quad$ S5

3. Determination of Kinetic Solubility of Cpds. 1-4, 30-31, 34, 36. S6

4. Metabolic Stability in Human Liver Microsomes (HLM) of Cpds. 1-

5. Reactivity with glutathione of Cpds. 1-4, 30-31, 34, $36 \quad$ S9

6. Analytical data for 2-aminooxazoles 7, 23-35, 50.

7. Analytical data for $N$-substituted-2-aminooxazoles 9, 24-31, 34 . S12

8. Figure $\mathrm{S} 1 \quad \mathbf{S 1 5}$

9. NMR for compounds 30, 31, 34, $36 \quad$ S16 


\section{Experimental}

Chemistry. All the reagents were purchased from Sigma-Aldrich, Alfa-Aesar, and Enamine at reagent purity and, unless otherwise noted, were used without any further purification. Dry solvents used in the reactions were obtained by distillation of technical grade materials over appropriate dehydrating agents. Microwave reactions were performed using CEM microwave synthesizer Discover model. Reactions were monitored by thin layer chromatography on silica gel coated aluminum foils (silica gel on Al foils, SUPELCO Analytical, Sigma-Aldrich) at both 254 and $365 \mathrm{~nm}$ wavelengths. When indicated, intermediates and final products were purified through silica gel flash chromatography (silica gel, $0.040-0.063 \mathrm{~mm}$ ), using appropriate solvent mixtures. ${ }^{1} \mathrm{H}$ NMR and ${ }^{13} \mathrm{C}$ NMR spectra were recorded on a BRUKER AVANCE spectrometer at 300, 400, and $100 \mathrm{MHz}$, respectively, with TMS as internal standard. ${ }^{1} \mathrm{H}$ NMR spectra are reported in this order: multiplicity and number of protons. Standard abbreviation indicating the multiplicity was used as follows: $\mathrm{s}=$ singlet, $\mathrm{d}=$ doublet, $\mathrm{dd}=$ doublet of doublets, $\mathrm{t}=$ triplet, $\mathrm{q}=$ quadruplet, $\mathrm{m}=$ multiplet and $\mathrm{br}=\mathrm{broad}$ signal. HPLC/MS experiments were performed with HPLC instrumentation (Agilent 1100 series, equipped with a Waters Symmetry C18, $3.5 \mu \mathrm{m}, 4.6 \mathrm{~mm} \times 75 \mathrm{~mm}$ column) and MS instrumentation (Applied Biosystem/MDS SCIEX, with API 150EX ion source). HRMS experiments were performed with LTQ ORBITRAP XL THERMO.

All compounds were tested as 95\% purity samples or higher (by HPLC/MS).

General procedure for the synthesis of 2-aminooxazoles 7, 17-23, 33 .

Conventional heating: Urea ( 2 - 10 equiv) was added to a solution of suitable $\alpha$-bromoketones ( 1 equiv) in dry DMF $(2,5 \mathrm{~mL} / \mathrm{mmol})$, and the reaction mixture was stirred at variable temperature (rt to $120^{\circ} \mathrm{C}$, see table 1) until the complete consumption of the starting material (as revealed by TLC, 95:5 dichloromethane/methanol) or, in alternative, for a total $18 \mathrm{~h}$. The reaction mixture was then quenched with water and the mixture extracted with ethyl acetate $(3 \times 10 \mathrm{~mL})$. The organic layers were washed with water, brine and dried over $\mathrm{Na}_{2} \mathrm{SO}_{4}$. After filtration, the solvent was removed in vacuo and the crude material was purified by flash column chromatography. 
Microwave irradiation: In a $10 \mathrm{~mL}$ test tube for microwave, urea (10 equiv) was added to a solution of $\alpha$-bromoketones ( 1 equiv) in dry DMF ( $3 \mathrm{~mL} / \mathrm{mmol})$, and the reaction mixture was irradiated in a microwave reactor after setting the parameters as follows: temperature $=120^{\circ} \mathrm{C}$, time $=3$ minutes, pressure $=250$ psi, power $=300 \mathrm{~W}$, power $\max =$ off. After the complete consumption of the starting material, as revealed by TLC (95:5 dichloromethane/methanol), water was added, and the mixture extracted with ethyl acetate $(3 \times 10 \mathrm{~mL})$. The organic layers were washed with water, brine and dried over $\mathrm{Na}_{2} \mathrm{SO}_{4}$. After filtration, the solvent was removed in vacuo and the crude material was purified by flash column chromatography.

The purification methods, yields, and the analytical data of the title compounds are reported in the following paragraphs.

General procedure for the Buchwald-Hartwig reaction. Synthesis of compounds 9, 24-31, 34. In a $10 \mathrm{~mL}$ test tube for microwave, a solution of the proper 2-aminooxazole (1 equiv), the suitable aryl halide ( 0.5 equiv), the base ( 1 equiv) in a mixture of anhydrous toluene $(2.5 \mathrm{~mL} / \mathrm{mmol})$ and $t$ butanol $(0.5 \mathrm{~mL} / \mathrm{mmol})$ was stirred under argon flux for 15 minutes. After this time, the suitable catalyst ( 0.1 equiv) was added and the reaction mixture was irradiated in a microwave reactor after setting the parameters as follows: temperature $=130^{\circ} \mathrm{C}$, time $=15$ minutes, pressure $=250$ psi, power $=300 \mathrm{~W}$, power $\max =$ off. The reaction was monitored by TLC. After the complete consumption of the starting material, as revealed by TLC (95:5 dichloromethane/methanol), water was added, and the mixture extracted with ethyl acetate $(3 \times 10 \mathrm{~mL})$. The organic layers were washed with water, brine and dried over $\mathrm{Na}_{2} \mathrm{SO}_{4}$. After filtration, the solvent was removed in vacuo and the crude material was purified by flash column chromatography.

The purification methods, yields, and the analytical data of the title compounds are reported in the following paragraphs.

5-(2-(p-tolylamino)oxazol-4-yl)isoxazole-3-carboxylic acid (35): Ester 34 (100 mg, $0.35 \mathrm{mmol})$ and $\mathrm{LiOH} \cdot \mathrm{H}_{2} \mathrm{O}(59 \mathrm{mg}, 1.4 \mathrm{mmol})$ were dissolved in a solution of $\mathrm{THF} / \mathrm{MeOH} / \mathrm{H}_{2} \mathrm{O}(3: 1: 1,1$ $\mathrm{mL} / \mathrm{mmol}$ ) and stirred at room temperature until consumption of the starting material as indicated by 
TLC (7:3 Petroleum ether/Ethyl Acetate, then 9:1 Dichloromethane/methanol). The reaction mixture is then evaporated under reduced pressure, and the crude obtained is taken up with $\mathrm{H}_{2} \mathrm{O}$, acidified with $2 \mathrm{~N} \mathrm{HCl}$ and extracted with ethyl acetate $(3 \times 10 \mathrm{~mL})$. After evaporation of the solvent, acid $\mathbf{3 5}$ is used for the next reaction step without further purification. (yield: $85 \mathrm{mg}$, quantitative). ${ }^{1} \mathrm{H}-\mathrm{NMR}$ (400 MHz, DMSO-d6) $\delta: 14.11(1 \mathrm{H}, \mathrm{bs}), 10.33(1 \mathrm{H}, \mathrm{s}), 8.40(1 \mathrm{H}, \mathrm{s}), 7.54(2 \mathrm{H}, \mathrm{d}, \mathrm{J}=8 \mathrm{~Hz}), 7.16(2 \mathrm{H}, \mathrm{d}$, $\mathrm{J}=8 \mathrm{~Hz}), 7.02(1 \mathrm{H}, \mathrm{s}), 2.27(3 \mathrm{H}, \mathrm{s}) ;{ }^{13} \mathrm{C}-\mathrm{NMR}(101 \mathrm{MHz}, \mathrm{DMSO}-\mathrm{d} 6) \delta: 165.1,161.1,158.1,157.8$, 136.9, 132.5, 131.0, 129.9, 128.6, 117.4, 101.7, 20.8. HRMS (ESI) analysis $[\mathrm{M}+\mathrm{H}]^{+}$: calculated for $\mathrm{C}_{14} \mathrm{H}_{11} \mathrm{~N}_{3} \mathrm{O}_{4}: 286.0750$; found: 286.08318

N-pyridinyl-5-(2-(p-tolylamino)oxazol-4-yl)isoxazole-3-carboxamide (36): $O$-(Benzotriazol-1yl)- $N, N, N^{\prime}, N^{\prime}$-tetramethyluronium tetrafluoroborate (TBTU, $96 \mathrm{mg}, 0.3 \mathrm{mmol}$ ) and $N$-(3Dimethylaminopropyl)- $N^{\prime}$-ethylcarbodiimide hydrochloride $(\mathrm{EDC} \cdot \mathrm{HCl}, 58 \mathrm{mg}, 0.3 \mathrm{mmol})$ were added to a solution of the carboxylic acid $35(85 \mathrm{mg}, 0.30 \mathrm{mmol})$ in dry DMF $(4 \mathrm{~mL} / \mathrm{mmol})$. The reaction mixture was stirred at room temperature under $\mathrm{N}_{2}$ for 15 minutes, then triethylamine (TEA, $62 \mu \mathrm{L}, 0.45 \mathrm{mmol})$ and 2-aminopyridine $(28 \mu \mathrm{L}, 0.3 \mathrm{mmol})$ were added to the mixture that was stirred at the same temperature until the complete consumption of the starting material, as indicated by TLC (7:3 Petroleum ether/Ethyl Acetate). Water $(10 \mathrm{~mL})$ was added and the mixture extracted with ethyl acetate $(3 \times 10 \mathrm{~mL})$. The organic layers were treated with water, washed with brine and dried over $\mathrm{Na}_{2} \mathrm{SO}_{4}$. After filtration, the solvent was removed in vacuo and the crude material was purified by flash column chromatography to give the amide 36 in 57\% yield as a yellow powder. ${ }^{1} \mathrm{H}-\mathrm{NMR}$ (400 MHz, DMSO-d6) $\delta: 10.73(1 \mathrm{H}, \mathrm{s}), 10.35(1 \mathrm{H}, \mathrm{s}), 8.44(1 \mathrm{H}, \mathrm{s}), 7.81(2 \mathrm{H}, \mathrm{d}, \mathrm{J}=8 \mathrm{~Hz}), 7.56(2 \mathrm{H}, \mathrm{d}, \mathrm{J}=8$ Hz), $7.39(2 \mathrm{H}, \mathrm{t}, \mathrm{J}=8 \mathrm{~Hz}), 7.19-7.14(4 \mathrm{H}, \mathrm{m}), 2.28(3 \mathrm{H}, \mathrm{s}) ;{ }^{13} \mathrm{C}-\mathrm{NMR}(101 \mathrm{MHz}, \mathrm{DMSO}-\mathrm{d} 6) \delta: 164.8$, 159.9, 158.1, 157.4, 138.5, 136.9, 132.5, 131.0, 129.9, 129.2, 128.6, 124.9, 121.1, 117.4, 101.0, 20.8. HRMS (ESI) analysis $[\mathrm{M}+\mathrm{H}]^{+}$: calculated for $\mathrm{C}_{19} \mathrm{H}_{15} \mathrm{~N}_{5} \mathrm{O}_{3}: 362.1175$; found: 362.13019 . 


\section{Determination of the MIC.}

For compound 36, MICs were determined following a previously reported method. ${ }^{1}$ Briefly, dilutions of M. tuberculosis wild-type cultures in exponential phase were streaked onto $7 \mathrm{H} 11$ solid medium containing a range of drug concentrations. Plates were incubated at $37^{\circ} \mathrm{C}$ for about 21 days and the growth was visually evaluated. The lowest drug dilution at which visible growth failed to occur was taken as the MIC value. For compounds 30, 31, and 34, MICs were determined using Mtb $\mathrm{H}_{37} \mathrm{Rv}$ ATCC 27294 in MABA and LORA assays according to published procedures. ${ }^{2,3}$ The reported MICs are an average value from 2-3 individual experiments. 


\section{Determination of Kinetic Solubility of compounds 1-4, 30-31, 34, 36.}

Kinetic solubility was determined starting from freshly prepared $10 \mathrm{mM}$ DMSO stock solutions of each test compound. In a 96-well plate, $2 \square \mathrm{L}$ of stock solution were added to $198 \square \mathrm{L}$ of either (i) water or (ii) $10 \mathrm{mM}$ PBS buffer $\mathrm{pH} 7.4$, adjusted to $0.15 \mathrm{M}$ ionic strength by $\mathrm{KCl}$ addition. Final DMSO in samples was $1 \% \mathrm{v} / \mathrm{v}$. The plate was stirred $(250 \mathrm{rpm}, 4 \mathrm{~h}$, room temperature). At the end of the incubation time, the precipitated compound was separated by centrifugation $(1000 \mathrm{~g}, 3 \mathrm{~min}$, $20^{\circ} \mathrm{C}$ ) and an aliquot of the supernatant was diluted 1:100 with $\mathrm{MeOH}$ and injected in the HPLCMS/MS system for quantification. Calibration curves for each test compound were built in the 1000$10 \mathrm{nM}$ concentration range starting from serial dilutions of DMSO standard solutions in $\mathrm{MeOH}$. 


\section{Metabolic Stability in Human Liver Microsomes (HLM) of compounds 1-4, 30-31, 34, 36.}

Xtreme human liver microsomes (HLM, pooled fraction derived from 200 male and female donors) were purchased from Xenotech, LLC (Cambridge, USA). Glucose-6-phosphate, $\beta$-nicotinamide adenine dinucleotide phosphate (NADP+), magnesium chloride, glucose-6-phosphatedehydrogenase were purchased from Sigma-Aldrich (St. Louis, USA). Briefly, a solution of enzymatic co-factors containing glucose-6-phosphate (10 mM), NADP+ (2 mM), $\mathrm{MgCl}_{2}(5 \mathrm{mM})$ and glucose-6-phosphate-dehydrogenase $(0.4 \mathrm{U} / \mathrm{mL})$ was prepared in $100 \mathrm{mM}$ phosphate buffered saline (PBS) pH 7.4. HLM suspension (i.e. $15 \mu \mathrm{L}$; final protein concentration $=1 \mathrm{mg} / \mathrm{mL}$ ) was added to 60 $\mu \mathrm{L}$ of co-factors mix and $222 \mu \mathrm{L}$ of PBS buffer. Samples were pre-incubated under continuous stirring at $37^{\circ} \mathrm{C}$ for $5 \mathrm{~min}$, then $3 \mu \mathrm{L}$ of DMSO stock solution of each test compound (final compound concentration $=1 \mu \mathrm{M}$; final DMSO concentration: $1 \% \mathrm{v} / \mathrm{v}$ ) were added to start the reaction. Sample aliquots were collected at different time points $(t=0,15,30,45,60 \mathrm{~min})$, enzymatic reactions were quenched by the addition of a double volume of acetonitrile $(\mathrm{ACN})$ containing a $100 \mathrm{nM}$ solution of internal standard (IS), samples were centrifuged (16000 g, $\left.10 \mathrm{~min}, 4{ }^{\circ} \mathrm{C}\right)$ and the supernatants were analyzed by HPLC-Multiple Reaction Monitoring (MRM)-MS for the amount of parent compound remaining. In vitro half-life (t1/2) and intrinsic clearance (CL'int) in liver subcellular fractions were calculated by the following equations: elimination rate constant $(\mathrm{k})=-$ slope; half-life $(\mathrm{t} 1 / 2) \min =$ ln2/k. Microsoft Excel (Microsoft Corp., 2010, USA) was employed for data analysis. GraphPad Prism v. 6.01 (GraphPad, USA) was employed for plotting graphs. Intrinsic Clearance values (CL'int) were calculated as follows: CL'int $(\mathrm{mL} / \mathrm{min} / \mathrm{kg})=\left(0.693 /\right.$ in vitro $\left.\mathrm{t}_{1 / 2}\right) \cdot($ incubation volume $/ \mathrm{mg}$ of microsomal protein $) \cdot(45 \mathrm{mg}$ microsomal protein/gram of liver $) \cdot(20 \mathrm{~g}$ of liver/kg body weight).

\section{HPLC-MS/MS analysis}

For the analysis of metabolic stability of compounds 1-4, 30-31, 34, 36 in HLM, LC-MS/MS analyses were carried out on a Thermo TSQ Quantum Access Max mass spectrometer (Thermo Fisher Scientific) interfaced to a Accela UHPLC system (Thermo Fisher Scientific) through a heated electrospray (H-ESI) ionization source. Chromatographic separation was conducted on a 
Phenomenex Synergy Fusion $80 \AA \AA$ column $(2.1 \times 100 \mathrm{~mm}$, i.d., $4 \mu \mathrm{m})$. Mobile phase consisted of $0.1 \%$ formic acid in water (A) and acetonitrile (B) with gradient procedures as follows: $0-1 \mathrm{~min} 5 \% \mathrm{~B}, 1-$ $6 \min 5-95 \%$ B,6-9 $\min 95 \% \mathrm{~B}, 9-10 \min 100 \% \mathrm{~B}, 10-11 \min 5 \% \mathrm{~B}, 11-145 \% \mathrm{~B}$. The flow rate was $0.35 \mathrm{ml} / \mathrm{min}$. ESI source was operated in positive and negative ion mode and in multiple reaction monitoring mode (MRM) with the optimized source parameters as follows: capillary voltage $3.5 \mathrm{kV}$ (ESI+) $2.5 \mathrm{kV}$ (ESI-); capillary temperature $270^{\circ} \mathrm{C}$; sheath gas (nitrogen gas) flow rate 35 arb; auxiliary gas (nitrogen gas) flow rate 15 arb. Argon with a pressure of 1.5 mtorr was employed as collision gas. Tube lens voltages (TL) and collision energies (CE) for each parent-product ion transition were optimized by Flow Injection Analysis (FIA) of $5 \mu \mathrm{M}$ solutions of each standard in MeOH. 1: $m / z=353.09[\mathrm{M}+\mathrm{H}]^{+} \rightarrow m / z=220.06,201.02,167.15[$ Tube Lens $(\mathrm{TL})=139 \mathrm{~V}$; Collision Energies $(\mathrm{CE})=35,37,38 \mathrm{eV}$, respectively); 2: $m / z=297.12[\mathrm{M}+\mathrm{H}]^{+} \rightarrow m / z=180.11,165.14,150.15$ $(\mathrm{TL}=111 \mathrm{~V} ; \mathrm{CE}=22,26,28 \mathrm{eV}) ; 3: m / z=330.00[\mathrm{M}+\mathrm{H}]^{+} \rightarrow m / z=235.10,217.06,189.11(\mathrm{TL}=$ $93 \mathrm{~V} ; \mathrm{CE}=13,21,26 \mathrm{eV}) ; 4: m / z=378.10[\mathrm{M}+\mathrm{H}]^{+} \rightarrow m / z=257.97,234.97,121.03(\mathrm{TL}=74 \mathrm{~V} ; \mathrm{CE}$ $=17,18,24 \mathrm{eV}) ; 30: m / z=281.09[\mathrm{M}+\mathrm{H}]^{+} \rightarrow m / z=148.10,133.24,121.34(\mathrm{TL}=118 \mathrm{~V} ; \mathrm{CE}=21$, $25,26 \mathrm{eV}) ; 31: m / z=337.06[\mathrm{M}+\mathrm{H}]^{+} \rightarrow m / z=258.99,190.02,177.03(\mathrm{TL}=55 \mathrm{~V} ; \mathrm{CE}=6,29,32$ $\mathrm{eV}) ; 34: m / z=314.13[\mathrm{M}+\mathrm{H}]^{+} \rightarrow m / z=215.05,118.15,91.33(\mathrm{TL}=101 \mathrm{~V} ; \mathrm{CE}=18,27,37 \mathrm{eV}) ; 36$ : $m / z=362.08[\mathrm{M}+\mathrm{H}]^{+} \rightarrow m / z=242.08,121.13,78.22(\mathrm{TL}=72 \mathrm{~V} ; \mathrm{CE}=16,22,36 \mathrm{eV}) ;$ Xcalibur software version 2.2 (Thermo, USA) was employed for both data acquisition and processing. 


\section{Reactivity with glutathione of compounds 1-4, 30-31, 34, 36}

$2 \mu \mathrm{L}$ of $2 \mathrm{mM}$ stock solution of test compounds in DMSO were added to $198 \mu \mathrm{L}$ of freshly-prepared 2 mM GSH solution (GSH: compound molar ratio 100:1) in 10 mM Phosphate Buffered Saline (PBS) buffer $\mathrm{pH} 7.4$, adjusted to $0.15 \mathrm{M}$ ionic strength by $\mathrm{KCl}$ addition. The formation of the corresponding GSH-conjugate was measured after $24 \mathrm{~h}$ of incubation at $37^{\circ} \mathrm{C}$ by HPLC-MS/MS acquiring in full scan mode $(\mathrm{m} / \mathrm{z}=100-800 \mathrm{amu})$ and in positive electrospray $(\mathrm{ESI}+)$ and extracting the single and double-charged ions corresponding to the GSH-conjugates of each compound.

Mobile phases were: A: ACN and B: ultra-pure water respectively, both containing $0.1 \% \mathrm{v} / \mathrm{v}$ formic acid. The following gradient was applied for the elution: A: ACN and B: ultra-pure water respectively, both containing $0.1 \% \mathrm{v} / \mathrm{v}$ formic acid, initial condition: $20 \% \mathrm{~A}$; linear gradient to $95 \% \mathrm{~A}$ in $12 \mathrm{~min}$; $95 \% \mathrm{~A}$ between 12 and 16 min returning to $20 \% \mathrm{~A}$ in $1 \mathrm{~min}$ with a 3 -min reconditioning time. Total run time: $20 \mathrm{~min}$. 
Analytical data for 2-aminooxazoles 7, 23-35, 50.

4-(p-tolyl)oxazol-2-amine (7) Purified by flash column chromatography, eluting petroleum ether/ethyl acetate 8/2. 66\% yield, ${ }^{1} \mathrm{H}-\mathrm{NMR}(300 \mathrm{MHz}$; DMSO-d6): $\delta=2.29 \mathrm{ppm}(\mathrm{s} ; 3 \mathrm{H}) ; 6.67 \mathrm{ppm}$ $(\mathrm{s} ; 2 \mathrm{H}) ; 7.15$ ppm (d; J = 6 Hz; 2H); 7.54 ppm (d; J = $6 \mathrm{~Hz} ; 2 \mathrm{H}) ; 7.80$ ppm (s; 1H). LRMS (ESI) calculated for $\mathrm{C}_{10} \mathrm{H}_{10} \mathrm{~N}_{2} \mathrm{O}[\mathrm{M}+\mathrm{H}]^{+} 175.08$ found 175.03

4-(o-tolyl)oxazol-2-amine (17) Purified by flash column chromatography, eluting petroleum ether/ethyl acetate 8/2. 52\% yield. ${ }^{1} \mathrm{H}-\mathrm{NMR}(300 \mathrm{MHz}$; DMSO-d6): $\delta=2.33 \mathrm{ppm}(\mathrm{s} ; 3 \mathrm{H}) ; 6.40 \mathrm{ppm}$ $(\mathrm{s} ; 2 \mathrm{H}) ; 7.28-7.38 \mathrm{ppm}(\mathrm{m} ; 3 \mathrm{H}) ; 7.46 \mathrm{ppm}(\mathrm{t} ; \mathrm{J}=6 \mathrm{~Hz} ; 1 \mathrm{H}) ; 8.40 \mathrm{ppm}(\mathrm{s} ; 1 \mathrm{H})$. LRMS (ESI) calculated for $\mathrm{C}_{10} \mathrm{H}_{10} \mathrm{~N}_{2} \mathrm{O}[\mathrm{M}+\mathrm{H}]^{+} 175.08$ found 175.04

4-(m-tolyl)oxazol-2-amine (18) Purified by flash column chromatography, eluting petroleum ether/ethyl acetate 8/2. 52\% yield. ${ }^{1} \mathrm{H}-\mathrm{NMR}(300 \mathrm{MHz}$; DMSO-d6): $\delta=2.41 \mathrm{ppm}(\mathrm{s} ; 3 \mathrm{H}) ; 5.86 \mathrm{ppm}$ $(\mathrm{s} ; 2 \mathrm{H}) ; 7.14$ ppm (s; 1H); 7.37-7.43 ppm (m; 2H); $7.69 \mathrm{ppm}(\mathrm{t} ; \mathrm{J}=8 \mathrm{~Hz} ; 1 \mathrm{H}) ; 8.40 \mathrm{ppm}(\mathrm{s} ; 1 \mathrm{H})$. LRMS (ESI) calculated for $\mathrm{C}_{10} \mathrm{H}_{10} \mathrm{~N}_{2} \mathrm{O}[\mathrm{M}+\mathrm{H}]^{+} 175.08$ found 175.03

4-(2-bromophenyl)oxazol-2-amine (19) Purified by flash column chromatography, eluting petroleum ether/ethyl acetate 8/2. 40\% yield. ${ }^{1} \mathrm{H}-\mathrm{NMR}(300 \mathrm{MHz}$; DMSO-d6): $\delta=6.81 \mathrm{ppm}(\mathrm{s} ; 2 \mathrm{H})$; 7.24 ppm (td; $\left.J_{1}=1.77 \mathrm{~Hz}, \mathrm{~J}_{2}=7.62 \mathrm{~Hz} ; 1 \mathrm{H}\right) ; 7.46-7.40 \mathrm{ppm}(\mathrm{m}, 1 \mathrm{H}) ; 7.69 \mathrm{ppm}\left(\mathrm{dd} ; ; \mathrm{J}_{1}=1.77 \mathrm{~Hz}\right.$, $\left.\mathrm{J}_{2}=7.62 \mathrm{~Hz} ; 1 \mathrm{H}\right) ; 7.93 \mathrm{ppm}\left(\mathrm{dd} ; \mathrm{J}_{1}=3 \mathrm{~Hz}, \mathrm{~J}_{2}=8 \mathrm{~Hz} ; 1 \mathrm{H}\right) ; 8.04 \mathrm{ppm}(\mathrm{s}, 1 \mathrm{H})$. LRMS (ESI) calculated for $\mathrm{C}_{9} \mathrm{H}_{7} \mathrm{BrN}_{2} \mathrm{O}[\mathrm{M}+\mathrm{H}]^{+} 238.97$ found 238.37

4-(3-bromophenyl)oxazol-2-amine (20) Purified by flash column chromatography, eluting petroleum ether/ethyl acetate $8 / 2.57 \%$ yield. ${ }^{1} \mathrm{H}-\mathrm{NMR}(300 \mathrm{MHz}$; DMSO-d6): $\delta=6.60 \mathrm{ppm}(\mathrm{s} ; 2 \mathrm{H})$; $7.12 \mathrm{ppm}(\mathrm{t} ; \mathrm{J}=8 \mathrm{~Hz} ; 1 \mathrm{H}) ; 7.41-7.46 \mathrm{ppm}(\mathrm{m}, 2 \mathrm{H}) ; 7.63 \mathrm{ppm}(\mathrm{t} ;$; J = $2 \mathrm{~Hz}, 1 \mathrm{H}) ; 8.40 \mathrm{ppm}(\mathrm{s}, 1 \mathrm{H})$. LRMS (ESI) calculated for $\mathrm{C}_{9} \mathrm{H}_{7} \mathrm{BrN}_{2} \mathrm{O}[\mathrm{M}+\mathrm{H}]^{+} 238.97$ found 238.03

4-(4-fluorophenyl)oxazol-2-amine (21) Purified by flash column chromatography, eluting petroleum ether/ethyl acetate 7/3. 53\% yield. ${ }^{1} \mathrm{H}-\mathrm{NMR}(400 \mathrm{MHz}$; DMSO-d6): $\delta=6.75 \mathrm{ppm}(\mathrm{s}, 2 \mathrm{H})$; 7.20 ppm $(\mathrm{d} ; \mathrm{J}=8 \mathrm{~Hz} ; 2 \mathrm{H}) ; 7.66 \mathrm{ppm}(\mathrm{d} ; \mathrm{J}=8 \mathrm{~Hz}) ; 7.86 \mathrm{ppm}(\mathrm{s} ; 1 \mathrm{H})$. LRMS (ESI) calculated for $\mathrm{C}_{9} \mathrm{H}_{7} \mathrm{FN}_{2} \mathrm{O}[\mathrm{M}+\mathrm{H}]^{+} 179.05$ found 178.95 
(22) Purified by flash column chromatography, eluting petroleum ether/ethyl acetate 7/3. 25\% yield. ${ }^{1} \mathrm{H}-\mathrm{NMR}$ (300 MHz; DMSOd6): $\delta=6.97 \mathrm{ppm}(\mathrm{s}, 2 \mathrm{H}) ; 7.56 \mathrm{ppm}(\mathrm{t}, \mathrm{J}=10 \mathrm{~Hz}, 1 \mathrm{H}) ; 7.71 \mathrm{ppm}(\mathrm{d}, \mathrm{J}=3 \mathrm{~Hz}, 1 \mathrm{H}) ; 7.86(\mathrm{~d}, \mathrm{~J}=3 \mathrm{~Hz}$, 1H); 8.00 ppm (s, 1H). LRMS (ESI) calculated for $\mathrm{C}_{10} \mathrm{H}_{6} \mathrm{~F}_{4} \mathrm{~N}_{2} \mathrm{O}[\mathrm{M}+\mathrm{H}]^{+} 247.04$ found 247.03 4-(4-methoxyphenyl)oxazol-2-amine (23) Purified by flash column chromatography, eluting petroleum ether/ethyl acetate 7/3. 38\% yield. ${ }^{1} \mathrm{H}-\mathrm{NMR}(300 \mathrm{MHz}$; DMSO-d6): $\delta=2.26 \mathrm{ppm}(\mathrm{s}, 3 \mathrm{H})$; $6.97 \mathrm{ppm}(\mathrm{s}, 2 \mathrm{H}) ; 7.58 \mathrm{ppm}(\mathrm{d}, \mathrm{J}=8 \mathrm{~Hz}, 2 \mathrm{H}) ; 7.70 \mathrm{ppm}(\mathrm{d}, \mathrm{J}=8 \mathrm{~Hz}, 2 \mathrm{H}) ; 8.00 \mathrm{ppm}(\mathrm{s}, 1 \mathrm{H}) . \mathrm{LRMS}$ (ESI) calculated for $\mathrm{C}_{10} \mathrm{H}_{10} \mathrm{~N}_{2} \mathrm{O}_{2}[\mathrm{M}+\mathrm{H}]^{+} 191.07$ found 191.37

Ethyl 5-(2-aminooxazol-4-yl)isoxazole-3-carboxylate (33) Purified by flash column chromatography, eluting petroleum ether/ethyl acetate $1 / 1.49 \%$ yield. ${ }^{1} \mathrm{H}-\mathrm{NMR}$ (400 MHz; DMSOd6): $\delta=1.35$ ppm (t, J = $8 \mathrm{~Hz}, 3 \mathrm{H}) ; 4.41 \mathrm{ppm}(\mathrm{q}, \mathrm{J}=8 \mathrm{~Hz}, 2 \mathrm{H}) ; 6.90 \mathrm{ppm}(\mathrm{s}, 1 \mathrm{H}) ; 7.07 \mathrm{ppm}(\mathrm{s}, 2 \mathrm{H})$; 8.16 ppm $(\mathrm{s}, 1 \mathrm{H})$. LRMS (ESI) calculated for $\mathrm{C}_{9} \mathrm{H}_{9} \mathrm{~N}_{3} \mathrm{O}_{4}[\mathrm{M}+\mathrm{H}]^{+} 224.06$ found 224.3 


\section{Analytical data for $N$-substituted-2-aminooxazoles 9, 24-31, 34.}

N-phenyl-4-(p-tolyl)oxazol-2-amine (9) Purified by flash column chromatography, eluting dichloromethane/methanol 96/4. 51\% yield. ${ }^{1} \mathrm{H}-\mathrm{NMR}\left(300 \mathrm{MHz}\right.$; DMSO-d $\left.{ }_{6}\right): \delta=2.33 \mathrm{ppm}(\mathrm{s}, 3 \mathrm{H})$; 6.95 ppm (m, 1H); 7.25 ppm (d, J = $6 \mathrm{~Hz}, 2 \mathrm{H}) ; 7.33$ ppm (t, J = $6 \mathrm{~Hz}, 2 \mathrm{H}) ; 7.72-7.66(\mathrm{~m}, 4 \mathrm{H}) ; 8.10$ ppm (s, 1H); 10.16 ppm (bs, 1H). HRMS (ESI) calculated for $\mathrm{C}_{16} \mathrm{H}_{14} \mathrm{~N}_{2} \mathrm{O}[\mathrm{M}+\mathrm{H}]^{+} 251,1106$ found 251,1098 .

N-(2-fluorophenyl)-4-(p-tolyl)oxazol-2-amine (24) Purified by flash column chromatography, eluting petroleum ether/ethyl acetate 97/3. 59\% yield. ${ }^{1} \mathrm{H}-\mathrm{NMR}\left(400 \mathrm{MHz}\right.$; DMSO-d $\left.{ }_{6}\right): \delta=2.33 \mathrm{ppm}$ $(\mathrm{s}, 3 \mathrm{H}) ; 7.01-7.06 \mathrm{ppm}(\mathrm{m}, 1 \mathrm{H}) ; 7.23-7.28(\mathrm{~m}, 4 \mathrm{H}) ; 7.66 \mathrm{ppm}(\mathrm{d}, \mathrm{J}=8 \mathrm{~Hz}, 2 \mathrm{H}) ; 8.13 \mathrm{ppm}(\mathrm{s}, 1 \mathrm{H})$; 8.32-8.44 (t, J = $8 \mathrm{~Hz}, 1 \mathrm{H}) ; 9.94$ ppm (bs, $1 \mathrm{H}) .{ }^{13} \mathrm{C}-\mathrm{NMR}\left(100.6 \mathrm{Mhz} ; \mathrm{DMSO}-\mathrm{d}_{6}\right): \delta=21.34 ; 115.65$; $115.85 ; 120.31 ; 122.94 ; 123.01 ; 125.33 ; 127.80 ; 128.46 ; 129.01 ; 129.67 ; 137.37 ; 139.42 ; 157.18$. HRMS (ESI) calculated for $\mathrm{C}_{16} \mathrm{H}_{13} \mathrm{FN}_{2} \mathrm{O}[\mathrm{M}+\mathrm{H}]^{+}$269,1012 found 269,1036.

N-(3-fluorophenyl)-4-(p-tolyl)oxazol-2-amine (25) Purified by flash column chromatography, eluting petroleum ether/ethyl acetate 97/3. 37\% yield. ${ }^{1} \mathrm{H}-\mathrm{NMR}(300 \mathrm{MHz}$; DMSO-d 6 ): $\delta=2.33 \mathrm{ppm}$ $(\mathrm{s}, 3 \mathrm{H}) ; 6.75-6.80 \mathrm{ppm}(\mathrm{m}, 1 \mathrm{H}) ; 7.24-7.26(\mathrm{~d}, \mathrm{~J}=6 \mathrm{~Hz}, 2 \mathrm{H}) ; 7.35-7.42 \mathrm{ppm}(\mathrm{m}, 2 \mathrm{H})$; 7.65-7.71 (m, 3H); 8.14 ppm (s, 1H); 10.45 ppm (bs, $1 \mathrm{H}) .{ }^{13} \mathrm{C}-\mathrm{NMR}\left(100.6 \mathrm{Mhz}\right.$; DMSO-d $\left.\mathrm{d}_{6}\right): \delta=21.34 ; 115.65$; $115.85 ; 120.31 ; 122.94 ; 123.01 ; 125.33 ; 127.80 ; 128.46 ; 129.01 ; 129.67 ; 137.37 ; 139.42 ; 157.18$. HRMS (ESI) calculated for $\mathrm{C}_{16} \mathrm{H}_{13} \mathrm{FN}_{2} \mathrm{O}[\mathrm{M}+\mathrm{H}]^{+}$269,1012 found 269,0998.

N-(4-fluorophenyl)-4-(p-tolyl)oxazol-2-amine (26) Purified by flash column chromatography, eluting petroleum ether/ethyl acetate $96 / 4.53 \%$ yield. ${ }^{1} \mathrm{H}-\mathrm{NMR}\left(400 \mathrm{MHz}\right.$; DMSO-d $\left.{ }_{6}\right): \delta=2.33 \mathrm{ppm}$ $(\mathrm{s}, 3 \mathrm{H}) ; 7.17-7.25 \mathrm{ppm}(\mathrm{m}, 4 \mathrm{H}) ; 7.67 \mathrm{ppm}(\mathrm{d}, \mathrm{J}=8 \mathrm{~Hz}, 2 \mathrm{H}) ; 7.72-7.74(\mathrm{~m}, 2 \mathrm{H}) ; 8.10 \mathrm{ppm}(\mathrm{s}, 1 \mathrm{H})$; 10.2 ppm (bs, 1H). ${ }^{13} \mathrm{C}-\mathrm{NMR}\left(100.6 \mathrm{Mhz} ; \mathrm{DMSO}-\mathrm{d}_{6}\right): \delta=21.34 ; 115.82 ; 116.04 ; 118.30 ; 118.37$; $125.31 ; 127.89 ; 129.65 ; 136.47 ; 137.32 ; 139.34 ; 157.17$. HRMS (ESI) calculated for $\mathrm{C}_{16} \mathrm{H}_{13} \mathrm{FN}_{2} \mathrm{O}$ $[\mathrm{M}+\mathrm{H}]^{+} 269,1012$ found 269,1070.

N-(2-methoxyphenyl)-4-(p-tolyl)oxazol-2-amine (27) Purified by flash column chromatography, eluting petroleum ether/ethyl acetate $97 / 3.71 \%$ yield. ${ }^{1} \mathrm{H}-\mathrm{NMR}\left(400 \mathrm{MHz}\right.$; DMSO-d $\left.{ }_{6}\right): \delta=2.33 \mathrm{ppm}$ 
(s, 3H); 3.85 ppm (s, 3H); 6.97-7.05 ppm (m, 3H); 7.24 (d, J = $8 \mathrm{~Hz}, 2 \mathrm{H}) ; 7.64$ ppm (d, J = $8 \mathrm{~Hz}$; 2H); $8.10 \mathrm{ppm}(\mathrm{s}, 1 \mathrm{H}) ; 8.26-8.27 \mathrm{ppm}(\mathrm{m}, 1 \mathrm{H}) ; 9.08 \mathrm{ppm}(\mathrm{bs}, 1 \mathrm{H}) .{ }^{13} \mathrm{C}-\mathrm{NMR}(100.6 \mathrm{Mhz}$; DMSO-d $): \delta$ $=\delta=21.34 ; 55.36 ; 103.00 ; 106.19 ; 109.55 ; 125.28 ; 127.89 ; 129.12 ; 129.69 ; 130.19 ; 137.31 ; 139.40$; 141.14; 157.03; 160.34. HRMS (ESI) calculated for $\mathrm{C}_{17} \mathrm{H}_{16} \mathrm{~N}_{2} \mathrm{O}_{2}[\mathrm{M}+\mathrm{H}]^{+}$281,1212 found 281,1190. N-(3-methoxyphenyl)-4-(p-tolyl)oxazol-2-amine (28) Purified by flash column chromatography, eluting petroleum ether/ethyl acetate 97/3. 59\% yield. ${ }^{1} \mathrm{H}-\mathrm{NMR}(400 \mathrm{MHz}$; DMSO-d 6 ): $\delta=2.33 \mathrm{ppm}$ (s, 3H); 3.77 ppm (s, 3H); 6.54-6.56 ppm (m, 1H); 7.21-7.25 (m, 4H); 7.45 ppm (s, 1H); 7.65 ppm $(\mathrm{d}, \mathrm{J}=8 \mathrm{~Hz}, 2 \mathrm{H}) ; 8.10$ ppm $(\mathrm{s}, 1 \mathrm{H}) ; 10.17 \mathrm{ppm}(\mathrm{bs}, 1 \mathrm{H}) .{ }^{13} \mathrm{C}-\mathrm{NMR}\left(100.6 \mathrm{Mhz} ; \mathrm{DMSO}-\mathrm{d}_{6}\right): \delta=$ $21.34 ; 55.36 ; 103.00 ; 106.19 ; 109.55 ; 125.28 ; 127.89 ; 129.12 ; 129.69 ; 130.19 ; 137.31 ; 139.40$; 141.14; 157.03; 160.34. HRMS (ESI) calculated for $\mathrm{C}_{17} \mathrm{H}_{16} \mathrm{~N}_{2} \mathrm{O}_{2}[\mathrm{M}+\mathrm{H}]^{+}$281,1212 found 281,1186. N-(4-methoxyphenyl)-4-(p-tolyl)oxazol-2-amine (29) Purified by flash column chromatography, eluting petroleum ether/ethyl acetate 97/3. 34\% yield. ${ }^{1} \mathrm{H}-\mathrm{NMR}\left(400 \mathrm{MHz}\right.$; DMSO-d $\left.{ }_{6}\right): \delta=2.33 \mathrm{ppm}$ (s, 3H); 3.73 ppm (s, 3H); 6.92 ppm (d, J=8 Hz; 2H); 7.24 (d, J = $8 \mathrm{~Hz}, 2 \mathrm{H}) ; 7.64$ ppm (m; 4H); 8.05 $\operatorname{ppm}(\mathrm{s}, 1 \mathrm{H}) ; 9.93 \mathrm{ppm}(\mathrm{bs}, 1 \mathrm{H}) .{ }^{13} \mathrm{C}-\mathrm{NMR}(100.6 \mathrm{Mhz}$; DMSO-d 6 ): $\delta=21.34 ; 55.67 ; 114.67 ; 118.38$; 125.38; 127.59; 129.26; 129.63; 133.41; 137.21; 139.37; 154.36; 157.18. HRMS (ESI) calculated for $\mathrm{C}_{17} \mathrm{H}_{16} \mathrm{~N}_{2} \mathrm{O}_{2}[\mathrm{M}+\mathrm{H}]^{+}$281,1212 found 281,1113 .

4-(2-fluoro-5-(trifluoromethyl)phenyl)- $N$-(p-tolyl)oxazol-2-amine (30) Purified by flash column chromatography, eluting petroleum ether/ethyl acetate 97/3. 31\% yield. ${ }^{1} \mathrm{H}-\mathrm{NMR}$ (300 MHz; DMSO$\left.\mathrm{d}_{6}\right): \delta=2.26 \mathrm{ppm}(\mathrm{s}, 3 \mathrm{H}) ; 7.18 \mathrm{ppm}(\mathrm{d}, \mathrm{J}=9 \mathrm{~Hz}, 2 \mathrm{H}) ; 7.53-7.62 \mathrm{ppm}(\mathrm{m}, 3 \mathrm{H}) ; 7.76-7.79 \mathrm{ppm}(\mathrm{m}$, 1H); 8.11 ppm (d, J = 4 Hz, 1H); 8.22 ppm (d, J = 4 Hz, 1H); 10.24 ppm (bs, $1 \mathrm{H}) .{ }^{13} \mathrm{C}-\mathrm{NMR}(100.6$ Mhz; DMSO-d $\left.{ }_{6}\right): \delta=21.3,116.3,120.4,123.8,124.5,126.6,127.1,128.1,129.8,131.2,135.9,139.9$, 140.2, 160.6, 161.9. HRMS (ESI) calculated for $\mathrm{C}_{17} \mathrm{H}_{12} \mathrm{~F}_{4} \mathrm{~N}_{2} \mathrm{O}[\mathrm{M}+\mathrm{H}]^{+} 337,0886$ found 337,0912. 4-(4-methoxyphenyl)- $N$-(p-tolyl)oxazol-2-amine (31) Purified by flash column chromatography, eluting petroleum dichloromethane/methanol 90/10. 10\% yield. ${ }^{1} \mathrm{H}-\mathrm{NMR}(400 \mathrm{MHz}$; DMSO-d $)$ : $\delta$ $=3.26 \mathrm{ppm}(\mathrm{s}, 3 \mathrm{H}) ; 3.79 \mathrm{ppm}(\mathrm{s}, 3 \mathrm{H}) ; 6.98 \mathrm{ppm}(\mathrm{d}, \mathrm{J}=8 \mathrm{~Hz}, 2 \mathrm{H}) ; 7.12-7.18 \mathrm{ppm}(\mathrm{m}, 1 \mathrm{H}) ; 7.58 \mathrm{ppm}$ $(\mathrm{d}, \mathrm{J}=8 \mathrm{~Hz}, 2 \mathrm{H}) ; 7.70 \mathrm{ppm}(\mathrm{d}, \mathrm{J}=8 \mathrm{~Hz}, 2 \mathrm{H}) ; 8.00 \mathrm{ppm}(\mathrm{s}, 1 \mathrm{H}) ; 9.98 \mathrm{ppm}(\mathrm{bs}, 1 \mathrm{H}) .{ }^{13} \mathrm{C}-\mathrm{NMR}(100.6$ 
Mhz; DMSO-d $\left.\mathrm{d}_{6}\right): \delta=111.51 ; 115.93 ; 116.14 ; 118.24 ; 121.08 ; 122.67 ; 125.95 ; 127.30 ; 127.38$; $128.39 ; 128.72 ; 138.53 ; 148.65 ; 157.63 ; 163.22$. HRMS (ESI) calculated for $\mathrm{C}_{17} \mathrm{H}_{16} \mathrm{~N}_{2} \mathrm{O}_{2}[\mathrm{M}+\mathrm{H}]^{+}$ 281,1212 found 281,1169 .

Ethyl 5-(2-(p-tolylamino)oxazol-4-yl)isoxazole-3-carboxylate (34) Purified by flash column chromatography, eluting petroleum ether/ethyl acetate 9/1. 16\% yield. ${ }^{1} \mathrm{H}-\mathrm{NMR}$ (400 MHz; DMSO$\left.\mathrm{d}_{6}\right): \delta=1.35 \mathrm{ppm}(\mathrm{t}, \mathrm{J}=8 \mathrm{~Hz}, 3 \mathrm{H}) ; 2.27 \mathrm{ppm}(\mathrm{s}, 3 \mathrm{H}) ; 4.41 \mathrm{ppm}(\mathrm{q}, \mathrm{J}=8 \mathrm{~Hz}, 2 \mathrm{H}) ; 7.10 \mathrm{ppm}(\mathrm{s}, 1 \mathrm{H}) ;$ 7.15-7.17 ppm (d, J = 2Hz, 2H); 7.53-7.55 (d, J = $8 \mathrm{~Hz}, 2 \mathrm{H}) ; 8.42$ ppm (s, 1H), 10.33 (bs, $1 \mathrm{H}) .{ }^{13} \mathrm{C}-$ NMR (100.6 MHz; DMSO-d $\left.{ }_{6}\right): \delta=14.42 ; 20.82 ; 62.45 ; 101.52 ; 117.39 ; 129.86 ; 131.05 ; 132.72 ;$ $136.99 ; 156.56 ; 136.99 ; 156.56 ; 158.31 ; 159.31 ; 159.35 ; 165.12$. HRMS (ESI) calculated for $\mathrm{C}_{16} \mathrm{H}_{15} \mathrm{~N}_{3} \mathrm{O}_{4}[\mathrm{M}+\mathrm{H}]^{+} 314,1063$ found 314,1112 . 


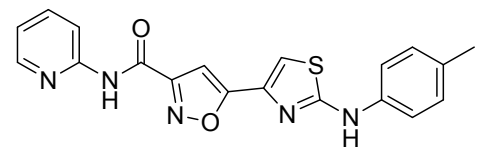

m/z: $378.1019[\mathrm{M}+\mathrm{H}]^{+}$

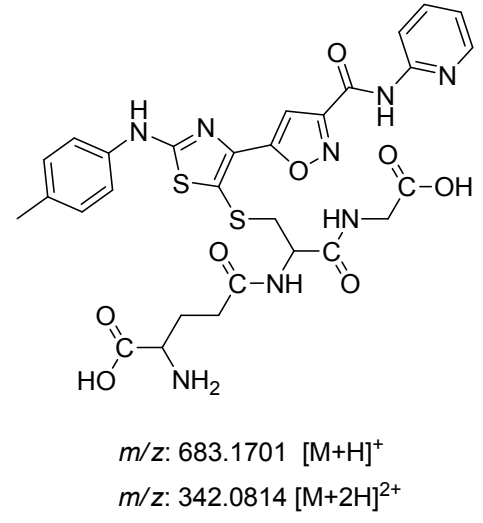

RT: 0.00 - 19.99 SM: 9G

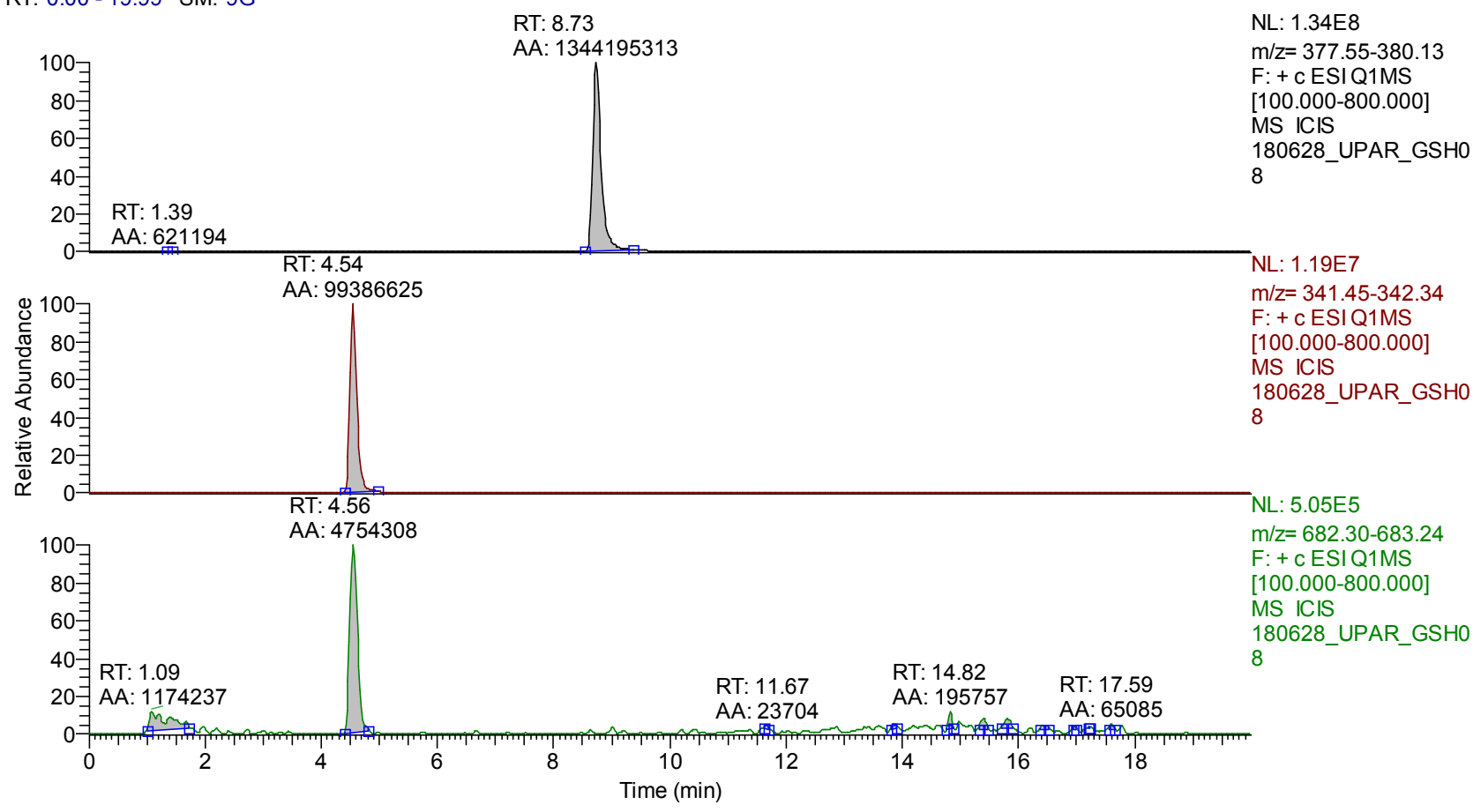

Figure S1. Example of HPLC-MS/MS ESI+ extracted ion chromatograms of a $24 \mathrm{~h}$ incubate of cpd.

4 with a 1:100 molar excess of GSH in PBS buffer, pH 7.4 at $37^{\circ} \mathrm{C}$. Trace \#1: Retention Time (RT) $=8.73 \mathrm{~min}$ and $\mathrm{m} / \mathrm{z}=378.10,[\mathrm{M}+\mathrm{H}]^{+}$ion corresponding to compound 4; Trace \#2: $\mathrm{RT}=4.54 \mathrm{~min}$ and $\mathrm{m} / \mathrm{z}=342.08,[\mathrm{M}+2 \mathrm{H}]^{2+}$ ion corresponding to 4-GSH conjugate; Trace \#3: RT $=4.56 \mathrm{~min}$ and $\mathrm{m} / \mathrm{z}=683.17,[\mathrm{M}+\mathrm{H}]^{+}$ion corresponding to 4-GSH conjugate. 
NMR of compounds tested 30, 31, 34, 36
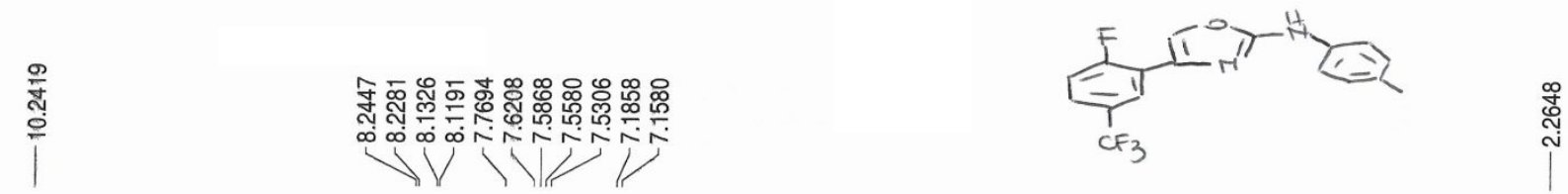

\section{Combound $\mathbf{3 0}$}

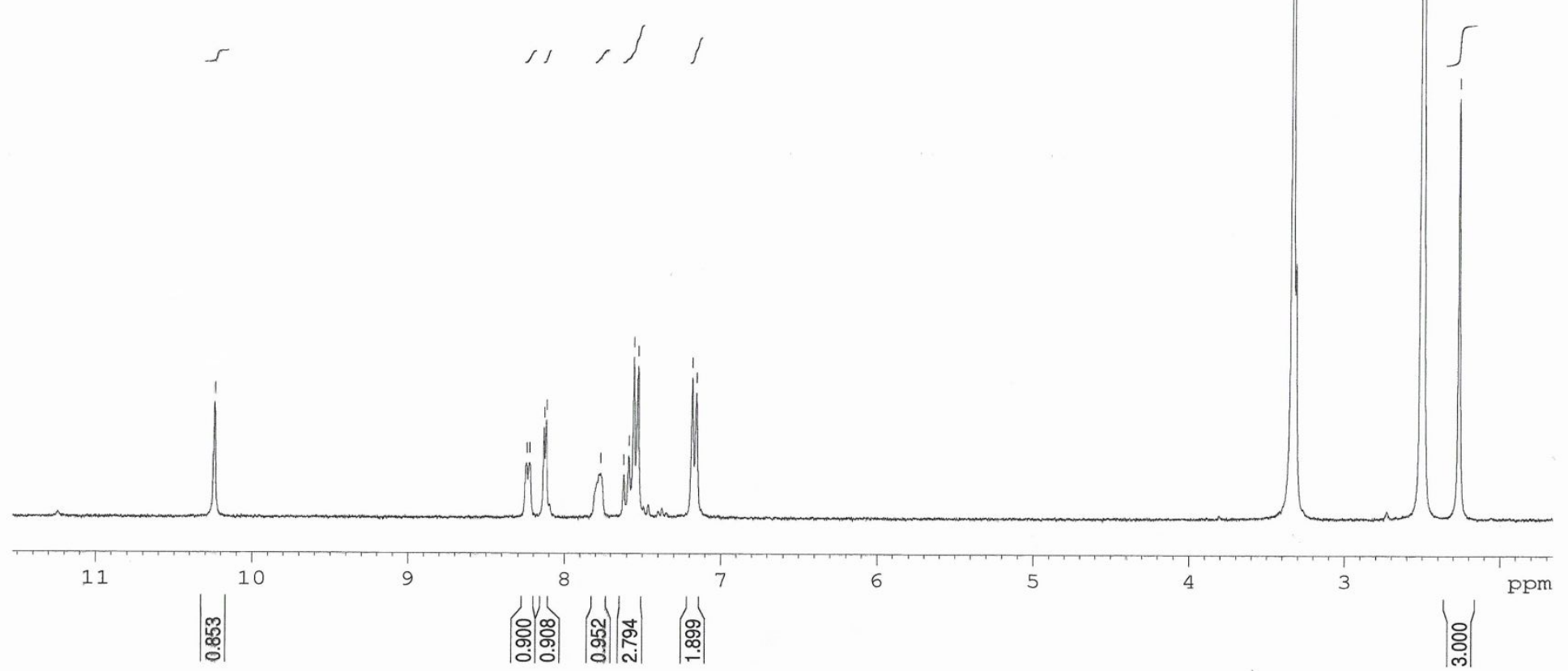




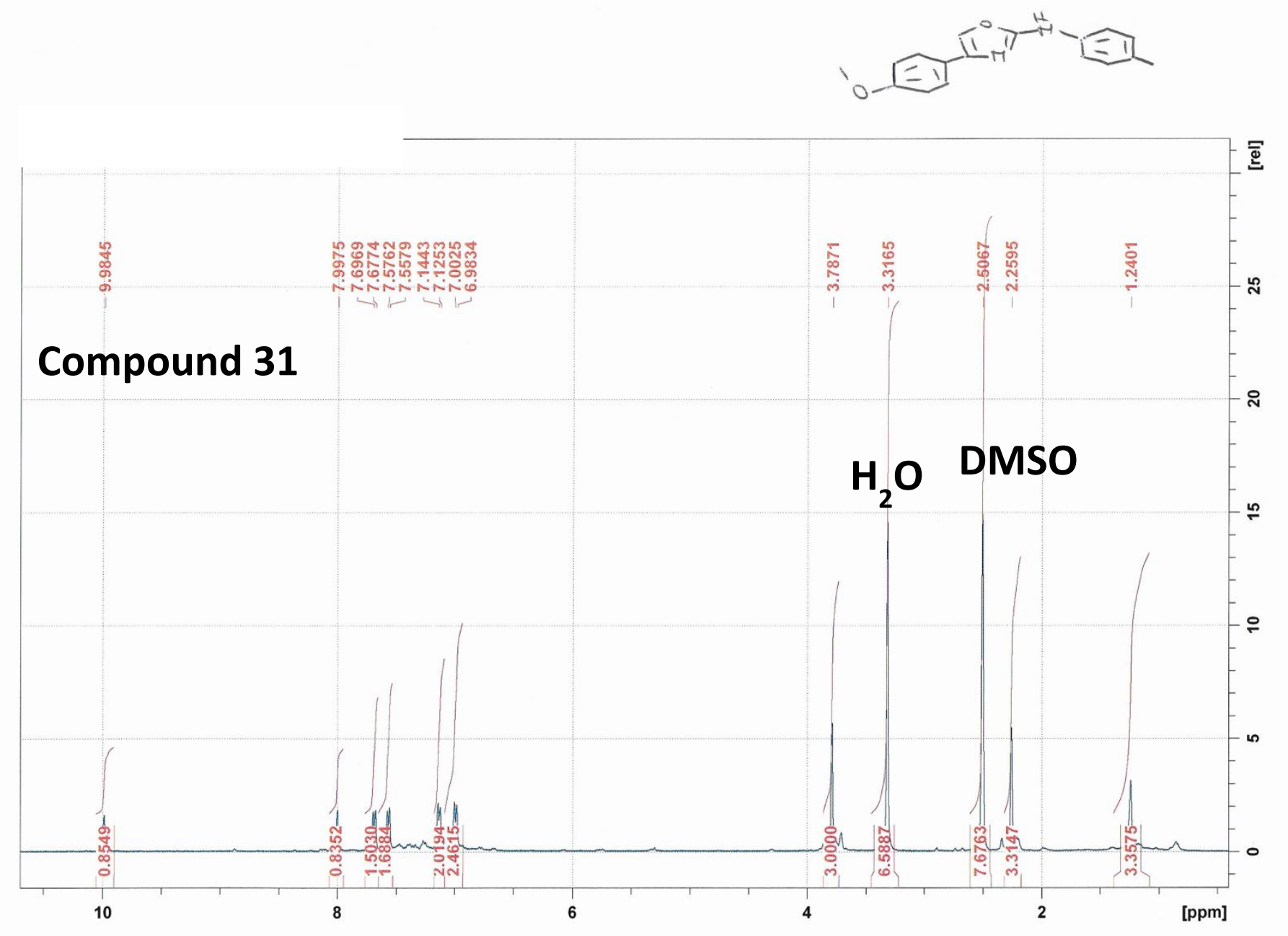




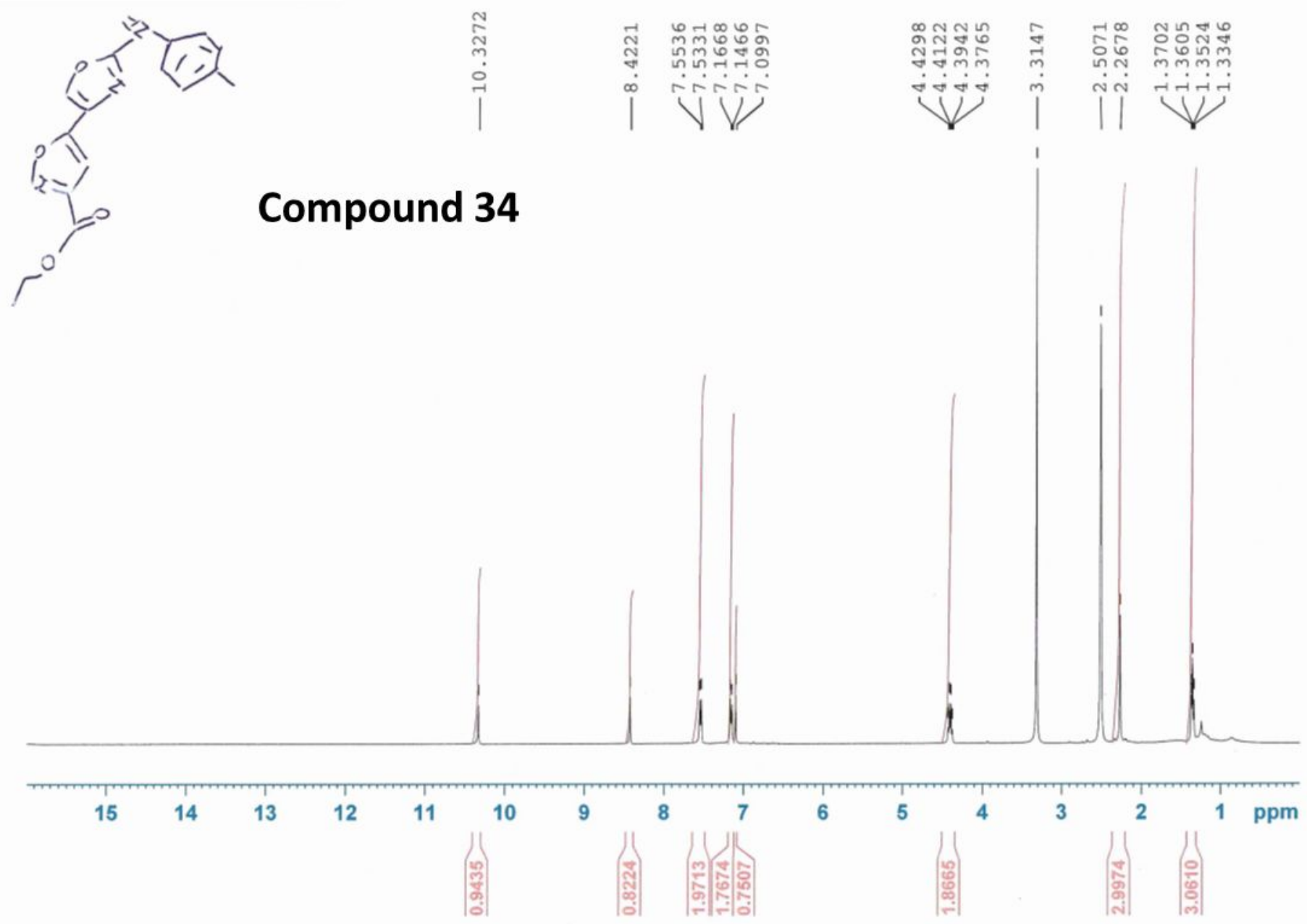




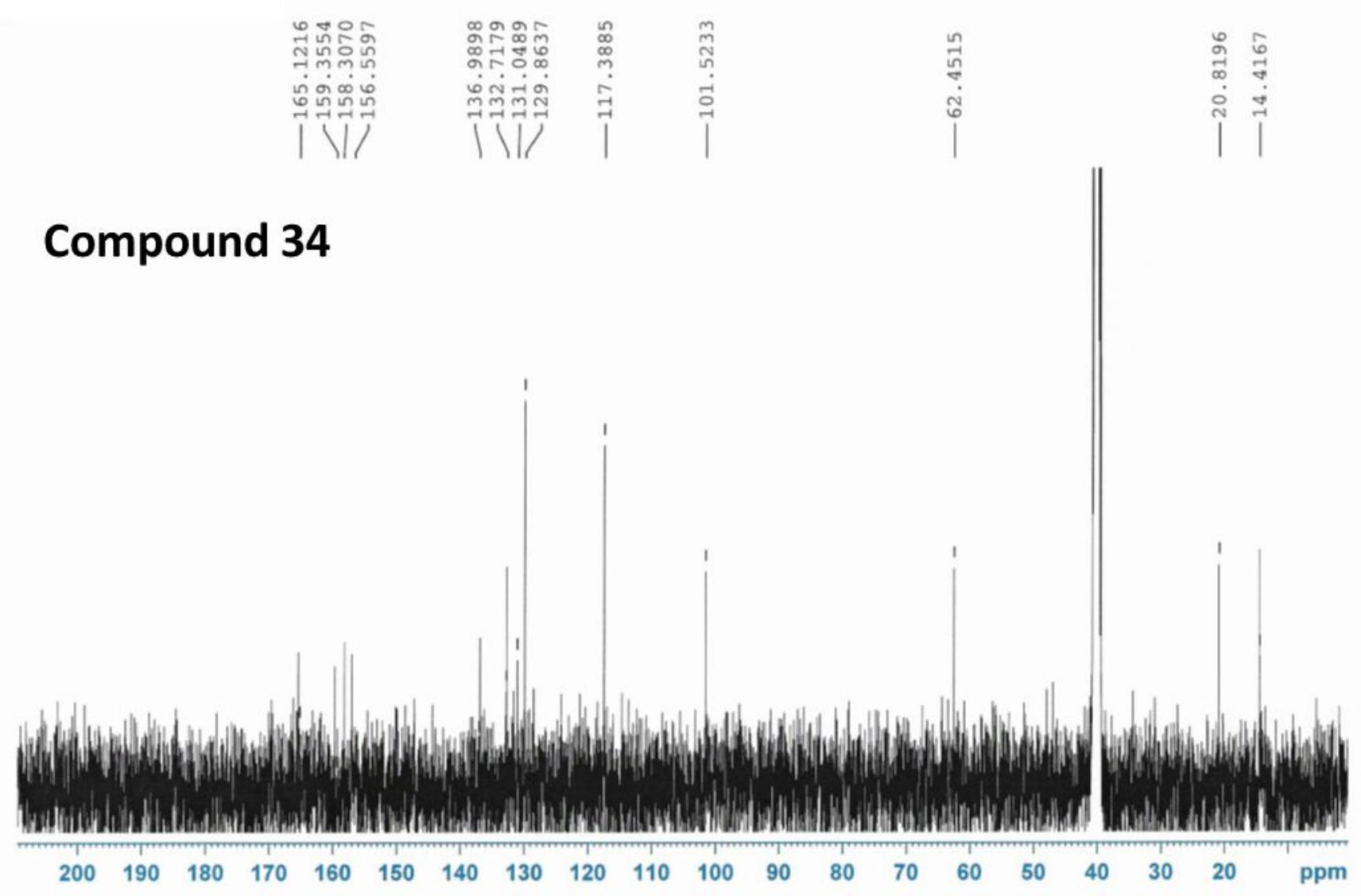




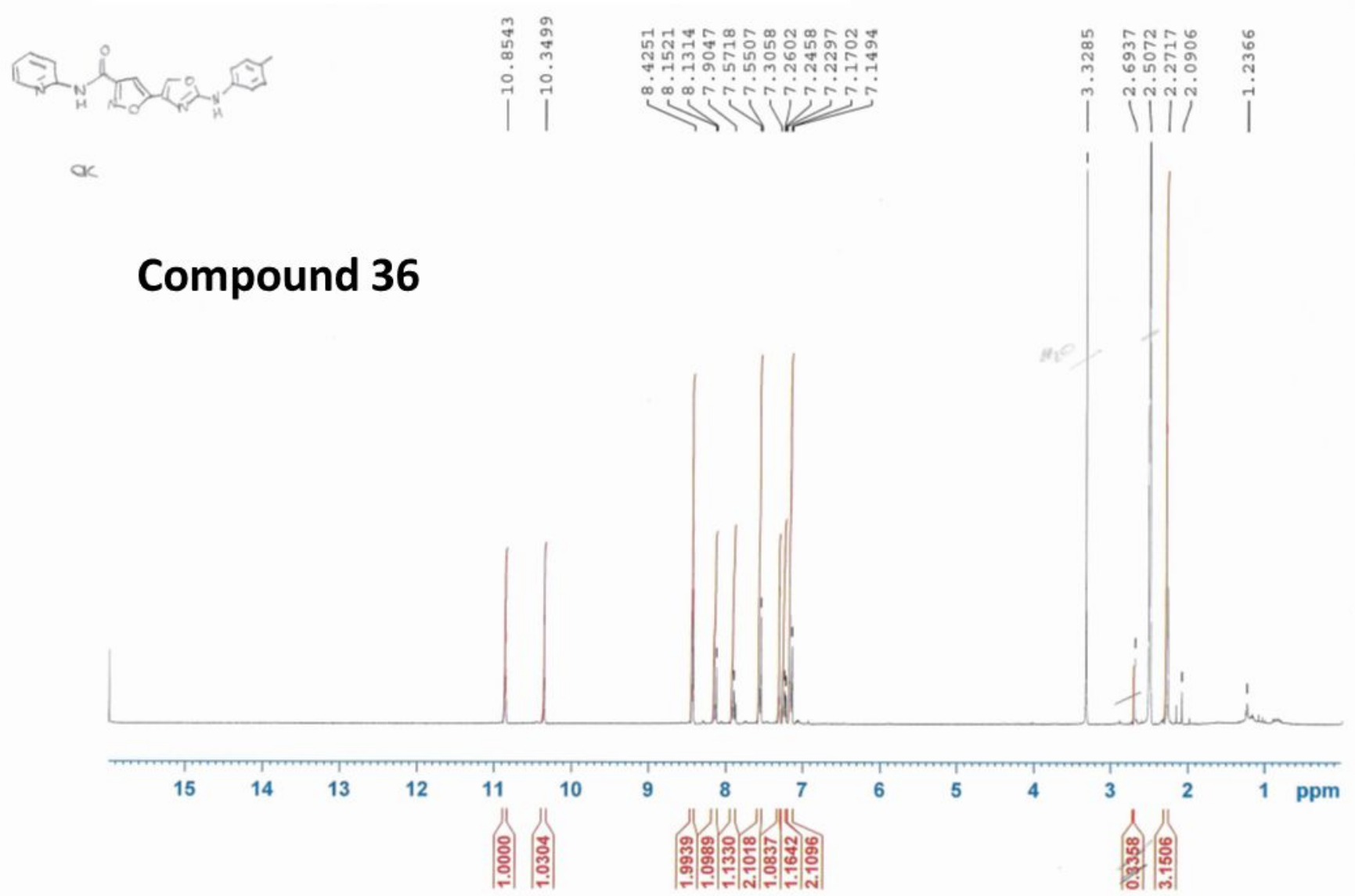


Compound 36

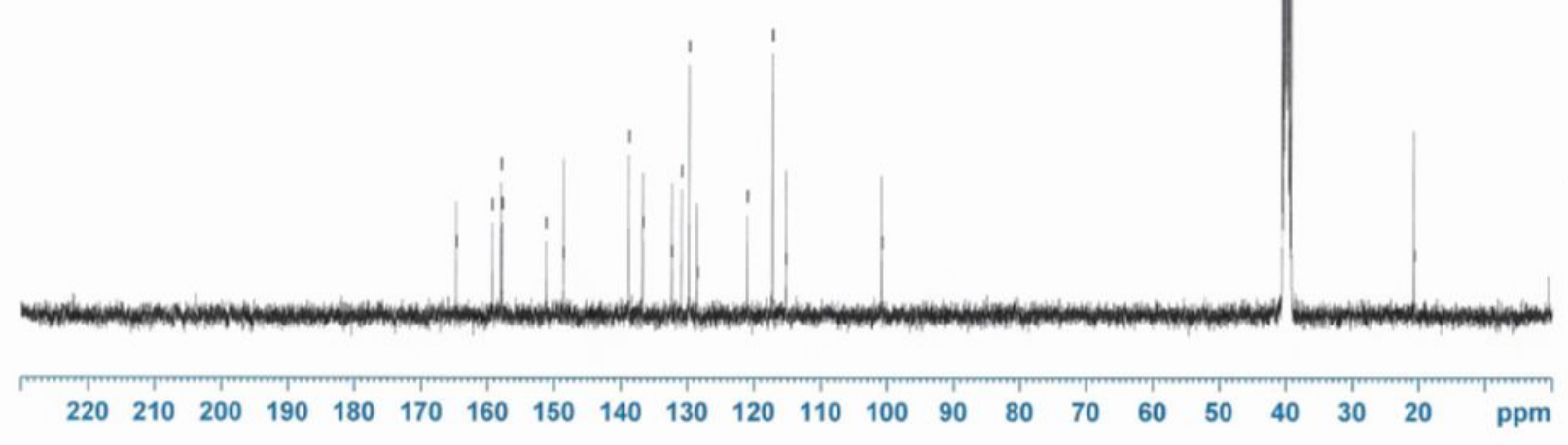

SI 


\section{References}

(1) Palomino, J.-C.; Martin, A.; Camacho, M.; Guerra, H.; Swings, J.; Portaels, F. Resazurin Microtiter Assay Plate: Simple and Inexpensive Method for Detection of Drug Resistance in Mycobacterium Tuberculosis. Antimicrob. Agents Chemother. 2002, 46 (8), 2720-2722. https://doi.org/10.1128/AAC.46.8.2720-2722.2002.

(2) Franzblau, S. G.; Witzig, R. S.; McLaughlin, J. C.; Torres, P.; Madico, G.; Hernandez, A.; Degnan, M. T.; Cook, M. B.; Quenzer, V. K.; Ferguson, R. M.; Gilman, R. H. Rapid, LowTechnology MIC Determination with Clinical Mycobacterium Tuberculosis Isolates by Using the Microplate Alamar Blue Assay. J. Clin. Microbiol. 1998, 36 (2), 362-366.

(3) Cho, S. H.; Warit, S.; Wan, B.; Hwang, C. H.; Pauli, G. F.; Franzblau, S. G. Low-OxygenRecovery Assay for High-Throughput Screening of Compounds against Nonreplicating Mycobacterium Tuberculosis. Antimicrob. Agents Chemother. 2007, 51 (4), 1380-1385. https://doi.org/10.1128/AAC.00055-06. 